\title{
Selective Mitophagy in budding yeast, a mitochondrial self-eating quality control
}

\section{Ziyad Tariq Muhseen}

Key Laboratory of Molecular Biophysics, Department of Biotechnology, College of Life Science and Technology, Huazhong University of Science and Technology, 1037 Luoyu Rd., Wuhan 430074, Hubei, China

\section{Email address:}

Ziyad.tariq82@gmail.com

\section{To cite this article:}

Ziyad Tariq Muhseen. Selective Mitophagy in Budding Yeast, a Mitochondrial Self-Eating Quality Control. European Journal of Biophysics. Vol. 2, No. 5, 2014, pp. 49-60. doi: 10.11648/j.ejb.20140205.11

\begin{abstract}
Mitochondria are responsible for the essential role in the cell survival by regulating the cellular energy, homeostasis, oxidative phosphorylation process, fatty acid oxidation and in the cell death process by regulating apoptosis. However, reactive oxygen species (ROS) is produced by mitochondria that pose oxidative damage to lipids, protein and mitochondrial DNA and additional production of ROS is triggered by this damage. On the other hand, the host cell control the life and death of mitochondria that include degradation, fission and growth. Recent research has been focused on the autophagic degradation of mitochondria, which is also mitophagy that results in significant discovery of the mechanism, function and regulation of mitophagy in eukaryotic cell especially in budding yeast. Mitophagy has been found to be important quality control mechanism for mitochondria. It has a key role in the eliminating of damaged mitochondria. Many studies have been done to unravel the mechanism and regulatory features of proteins involved in mitophagy but the results are inconsistent and conflicting. Mitochondrial surface receptor involved in mitochondrial autophagy has been recently identified using yeast genetics. Recent studies have been discovered specific regulators of Mitophagy that ensure selective sequestration of mitochondria as cargo. According to our understanding, in this paper we will review recent advances of different pathways of Mitophagy in the yeast. We will review the main proteins which play an essential role in controlling this process and the main pathways which lead to a highly controlled Mitophagy process.
\end{abstract}

Keywords: Mitochondria, Mitophagy, Yeast, Autophagy

\section{Author Summary}

Autophagy, a developmental process in eukaryotic cell, transports cytoplasmic contents for degradation into the lysosomes. It relates to multiple human diseases, including neurodegenerative disorders and cancer. Saccharomyces cerevisiae, the first organism in which molecular machinery of autophagy has been best characterized but in other unicellular model organism, a little knowledge is known for the molecular machinery of autophagy.

Mitochondria, as a highly dynamic organelle, undergo and suffer constitutive fusion and fission as well as degradation and biogenesis. Mitophagy, the selective mitochondrial degradation process through autophagy, is a conserved cellular process which used for the elimination of damaged and excessive mitochondria in eukaryotes. Although the significance of mitophagy in cellular pathophysiology and physiology, the basic mechanism of this process is far from clear. For the maintenance of cellular homeostasis, the control of number and quality of mitochondria is very important. Mitochondria have the most important function in the cell. By carrying out metabolic processes such as oxidative phosphorylation and oxidation of fatty acid, mitochondria provide the cell with huge amount of energy. On the other hand, the mitochondria are involved in the production of harmful ROS which damages proteins, lipids and DNA. As a result the accumulation of damage macromolecule molecules cause neurodegeneration, aging and cancer. Yeast cell when growing under normal condition may face nutritional changes that require the cell to adopt their mitochondrial contents. For example the change from lactate to fermentative one respiratory carbon source (e.g. glucose) results in decrease of enzymatic activity of mitochondria that is achieved by a decrease in the number of mitochondria. To regulate the number of mitochondria, one of the main mechanisms is to inhibit its biogenesis. However, 
for the fast adaptation the turnover of the existing mitochondria is required. Studies that have done on both the mammalian cells and yeast indicate that a crucial rule will play by autophagy in many catabolic processes. In addition, to regulate the number of mitochondria, the recognition and clearance of damage mitochondria are necessary for cell survival because they release the chemical factors which cause cell death such as cytochrome c, reduce the efficiency of ATP generation and produce ROS in an amount which is toxic.

\section{Autophagy in Mitochondria}

Autophagy, a conserved catabolism in almost all eukaryotes, is responsible for the degradation of cytoplasmic components and organelles [1]. Diverse physiological processes, which are commenced in response to cellular stresses such as oxidative stress, nutrient starvation, inflammatory stimuli and infection, are controlled by autophagy [2]. Upon the induction of above cellular stresses a phagophore is appeared in cytoplasm, which is a double membrane structure and also called isolation membrane, followed by the elongation of isolation membrane forming autophagosomes enclosing cytoplasmic components. Throughout this process, the luminal side of autophagosomal inner vesicle formed by the concave side of growing phagophore and finally it gets enclosed containing cytoplasmic components forming and auto-phagosome body [3-5]. Subsequently, the fusion of auto-phagosome with lysosomes will occurred that results in the degradation and hydrolysis of sequestered material (Figure 1) [6].

This process is responsible to accelerates physiological processes such as the survival of cell during starvation, clearance and removal of dysfunctional or aggregated proteins and organelles, differentiation, aging and development $[7,8]$.

The template is used to format your paper and style the text. All margins, column widths, line spaces, and text fonts are prescribed; please do not alter them. You may note peculiarities. For example, the head margin in this template measures proportionately more than is customary. This measurement and others are deliberate, using specifications that anticipate your paper as one part of the entire publication, and not as an independent document. Please do not revise any of the curr

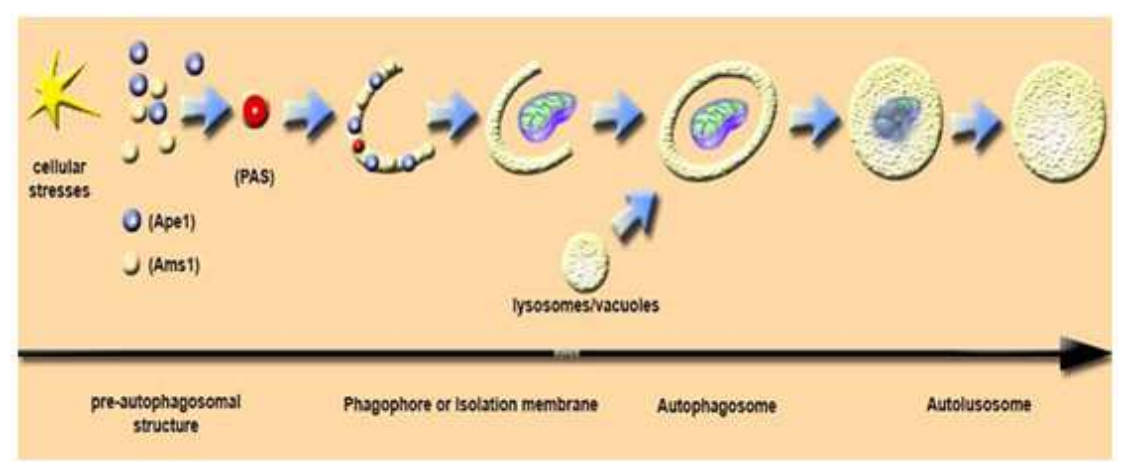

Figure 1. Schematic of the process of Autophagy in yeast

A protein complex in the yeast (Cvt complex) containing amino peptidase I (Ape1) and apha-mannosidase (Ams1) delivered to vacuole through an autophagy. Subsequently the Ape1 and Asm1 are activated due to processing in vacuole. This process is known as Cvt pathway. Clark et al. in 1957 [9] and Takeshige et al. 1992 [10] for the first time described that mitochondria are also degraded in mammalians cells and in yeast respectively by process of autophagy. Since then, it thought that during macro-autophagy the mitochondria are engulfed non-selectively as a part of cytoplasm by autophagosomes. Recently, the engulfment of mitochondria through a selective autophagy has been suggested by some researcher in detail [11-13]

In yeast cell, after fission event the new born mitochondria with reduced membrane potential are removed by autophagy $[14,15]$. The autophagy process will remove damaged mitochondria selectively [16]. Similarly mitochondria are degraded by autophagy during adipose tissue differentiation [17]. When yeast cells are cultured in a medium containing lactate as source of carbon and then deprived from nitrogen the mitochondria are converted into micro-autophagic structure [11]. This support the idea that autophagy is involved in recognition and selective degradation of mitochondria. The selective degradation of mitochondria was further confirmed by the identification of mitophagy-specific protein (Atg32) of yeast. The Atg32 has been discovered to be important for the recognition of mitochondria by autpohagic machinery [18, 19].

Two types of autophagy exist in yeast cell; one is the autophagy related protein 32 (Atg32)-dependent autophagy (conventional) and second is Atg32 independent autophagy (alternative) [20]. In the autophagic degradation of mitochondria both the conventional and alternative processes are involved but the Atg32 dependent autophagy plays an important role as mitophagy receptors [20-22]. The absence of Atg32 protein will block the process of Mitophyagy and its overexpression induces the process [22].

\subsection{Mitophagy: One Part of Selective Autophagy in Yeast}

In eukaryotic cells, there are two major degradation process pathways; Autophagy and ubiquitin-proteasome system. The main function of the autophagy is the degradation of the entire 
organelles and long live proteins [23]. In the yeast cell most of the organelles and macromolecules complexes are degraded by macro-autophagy and micro-autophagy. In macro-autophagy, a double membrane auto-phagosome is formed which seize the macro molecular complex. The auto-phagosome then fuses with the lysosome and start the degradation of the cargo. On the other hand micro-autophagy, which is a related process to macro-autophagy, in which the direct engulfing of cytosolic materials (Figure 2) occurred by membrane limiting vacuole [23].

The ubiquitin-proteasome system (UPS) which is responsible for the degradation of the majority of the proteins (80-90\%), including short live proteins, regulated proteins, denatured proteins, abnormal and damaged proteins [24, 25].

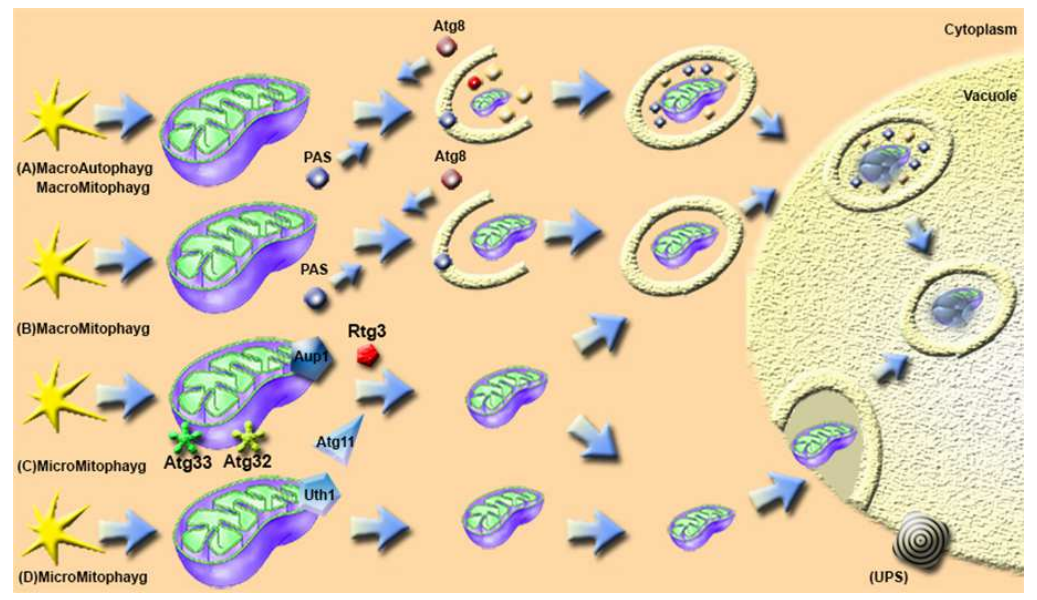

Figure 2. Schematic of the Overview of Autophagy and Mitophagy in Yeast (a) Macro-autophagy, Atg proteins (Atg8, Red dots), forming the (PAS) and sequesters cytoplasmic components into Autophagosomes then deliver it into the vacuole for degradation. (b) Macro-mitophagy involves the vacuolar membrane in order to deliver cytoplasmic contents for degradation. (c) Mitochondria can be selectively degraded through a Micro-autophagic mechanism. This requires the activity of Atg32, Atg33 and Atg11p to bring the selected mitochondria into contact with the core autophagy machinery; Mitochondria can be removed by selective Micro-autophagy, or Micro-mitophagy, the mechanism of which remains unclear. While Atg32 and Atg11p may be involved in Micro-mitophagy, Aup1 and Rtg3 function remains undetermined. (d) Mitochondria can also be removed by selective Micro-autophagy, or Micro-mitophagy, Uth1 be involved in the process. The ubiquitin-proteasome system (UPS)

There are two types of autophagy, the selective and non-selective. In the non-selective autophagy, the auto-phagosome randomly sequestered the bulk cytosol and other cytoplasmic components of the cell. On the other hand, in selective auto-phagosome the specific cargo is sequestered by a double membrane vesicle. The vesicle adopts the shape of cargo and this structure contains a little cytoplasm. Selective autophagy uses double membrane vesicle to transport cytoplasmic pathogens, cytoplasmic damaged organs, organelles and protein complexes to the vacuole for degradation. The conserved core autophagy machinery is being used in all selective autophagy and in the elimination of specific cargo; the selective autophagy should keep a mechanism that assures the accurateness of elimination of specific cargo.

The main important issue for the maintenance of cellular homeostasis is the control of number and quality of mitochondria. The primary source of energy is the mitochondria which generate energy by carrying out important metabolic processes such as the oxidation of fatty acid and the oxidative phosphorylation. However the harmful ROS is also produce by mitochondria as the side product during oxidative phosphorylation which damage lipids, DNA and proteins. The accumulation of these damaged molecules is implicated in process of aging, neurodegeneration and cancer [26, 27]. The yeast cell faces nutritional changes when grown in under natural condition. To overcome this problem the cell required to adjust their content of their mitochondria
$[28,29]$. For example the change from lactate to fermentative respiratory carbon source (e.g. glucose), results in decrease of enzymatic activity of mitochondria that is achieved by a decrease in the number of mitochondria. To regulate the number of mitochondria, one of the main mechanisms is to inhibit its biogenesis. However, for the fast adaptation the turnover of the existing mitochondria is required.

Studies have shown that autophagy is involved in major catabolic events. Regulation of the number of mitochondria is not only the task performed by mitophay but it also have important role to recognize and clear the damage and useless mitochondria. This process is very important for the cell's survival as the damage mitochondria release the factor (cytochrome c) that cause cell death and cause reduction in the efficiency of generation of ATP. The damage mitochondria also produce ROS in toxic amount [30].

In 1977 the observation of selective degradation of mitochondria by autophagy was made by studying the metamorphosis of insect [31]. In 1992, mitochondria has discovered in the interior portion of autophagosomes of yeast cell that has deprived from nitrogen (nitrogen starvation) [10]. Further studies have confirmed that the vesicle in the vacuole or autophagic bulks contain both the mitochondria and cytoplasm or either mitochondria and cytoplasm. The later studies have shown that mitochondria are sequestered selectively by autophagosomes in yeast cell. To date, the degradation of mitochondria by selective type of autophagy has been supported by several studies and term as mitophagy. 


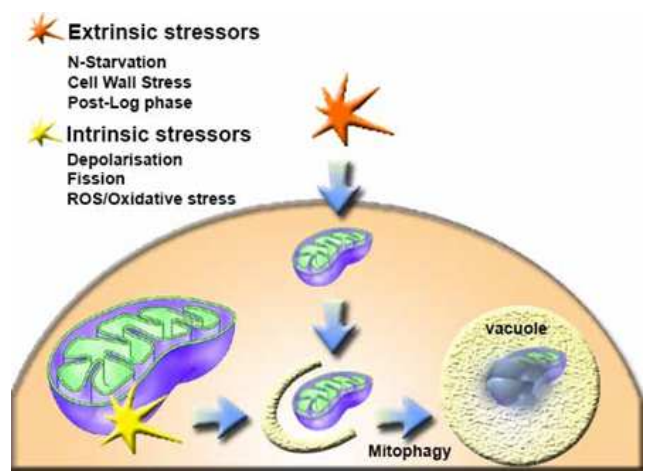

Figure 3. Mitophagy response to stress. Stress signals (extrinsic red colour from outside the cell) and (extrinsic red colour from outside the cell) or (intrinsic yellow colour from inside the cell), the mitochondrion, interact with regulatory intermediates in the cell, promoting the removal of excess or damaged mitochondria. As a consequence, mitochondria are then removed by an Autophagic mechanism, Mitophagy.

\subsection{Mitophagy Mechanisms}

To gain insight into the mitophagy, a number of different mechanisms have been described such as selective micro-autophagy [32], Non-specific macro-autophagy [10], selective macro-autophagy $[11,33,34]$. Still the fundamental mechanism of mitophagy is largely not clear. Nevertheless, two proteins that are essential for the mitophagy in yeast have been characterized. A mitochondrial phosphatase (Aup1), present in the inner folded mitochondrial membrane space, is essential for the mitophagy that is induced during the stationary phase of cell [35]. The deletion of Aup1 effects the normal growth and development of cell during the beginning of mitophagy induction. Though, the available data shows that it tends to be selective micro-autophagy but it is largely unclear whether mitophagy occur in this condition is macro-autophagy or micro-autophagy (Figure 2.C) [36]. Uth1, essential for mitophagy, is another protein resides in the outer membrane of mitochondria. Uth1 is required during starvation for the effective mitophagy (Figure 2.D) [35]. Unlike Aup1 (deletion of which decrease cell viability), the deletion of Uth1 increases cell growth and life span during nitrogen starvation. The observation under electron microscopy showed that mitochondria are swept by micro-autophagy in the yeast cell when grown in a culture with lactate (non-fermentable carbon source) and in the absence of nitrogen source [11]. These observations further clarified that two types of micro-autophagic structures that contain mitochondria were observed; the autophagic bodies either contain mitochondria or mitochondria and cytoplasm. The existence of both selective and non-selective autophagy were suggested by the above two observation whereas the selective pathway only depend on Uth1. In non-selective macro-autophagy none of Aup1 and Uth1 is required, supporting the idea of a selective Mitophagy process pathway. Though both proteins are required for mitophagy, the knockout of Uth1 increases the cell viability under mitophagy induced condition, whereas the knockout of Aup1 decrease the cell viability thus cells with Aup1 are sensitive to rapamycin where as Uth1 mutant cells are highly resistant to the to the same toxin. However these data should be carefully deduced because different degradation pathways were triggered by the use of different experiments to eliminate mitochondria, induced starvation against long term stationary phase. Furthermore, these two proteins may have function in cell other than mitophagy that are responsible for different phenotype. Due to lack of knowledge about functional interaction between Aup1 and Uth1, the mechanistic model about mitophagy that integrate these two proteins could not be drawn. Interestingly, Uth1 protein is regulated by phosphorylation [35], but it looks unlikely that Aup1 is responsible for its dephosphorylation because these two proteins are spatially separated.

Further studies have shown that during the stationary phase and starvation, the mitophagy depends upon a gene (ATG11) responsible for autophagy of selective types such as pexophagy and Cvt pathway but not essential for non-selective autophagy [37-39], this finding support the existence of selective type of mitophagy [37].

The cell with mutant Aup1 make the strain deficient of autophagy and were unable to degrade the mitochondria during a stationary phase and cause defect in growth and the cell remains in the G1 phase of the cell cycle. Furthermore, a high amount of ROS is produced by the mutant cell and accumulation of dysfunctional mitochondria occurred in mutant cell. Thus it suggests that cells capable of carrying autophagy are able to degrade damage mitochondria selectively [38].

There is a controversy between the role of autophagy and mitophagy in the cell survival process. In Saccharomyces cerevisiae, under certain condition, the process of mitophagy often correlates with cell death phenomenon [33, 40]. The central regulator of cell death in mammalian cells is mitochondria and the loss of its membrane potential initiate apoptosis and other diseases [27, 41-43].

A recent study has shown that Parkin (a protein that is responsible for the initiation of Parkinson's disease) engaged selectively to dysfunctional mitochondria and causes their degradation through autophagy. This suggests the failure to sweep the damage mitochondria out of cell during pathogenesis of Parkinson's disease [44]. In addition, any defect or damage to mitochondria account for cancer and aging $[45,46]$.

As the damage of nuclear and mitochondrial DNA is caused by the production of ROS, but unlike to nucleus the mitochondria do not have DNA damage repairing and protection system so, mitochondrial DNA has more chance of mutations, resulting in a $10-1000$ fold higher mutation rate than nuclear DNA [45]. On the contrary, cancer cells typically show high levels of mitochondrial mutations and dysfunctions [47, 48].

The aging phenomenon is also concerned with damaged DNA and non-functional components accumulation. Consequently, mitophagy play a vital role to maintain cell health and to prevent aging by sweeping damaged mitochondria out of cell [9]. 


\subsection{Autophagosomes Formation in Mitophagy}

Autophagosomes are formed de novo by membrane fusion events at the phagophore assembly site (PAS). Therefore, primary precursor membrane material must be targeted and transported to the PAS. While some autophagy-related (Atg) proteins, such as Atg8, Atg9 and Atg11, involve in this process, most of the mechanistic details are not understood $[49,50]$ (Figure 1).

In this study, we review sensitive and convenient proteins to monitor Mitophagy. This means that selective degradation of mitochondria occurs in different manner from bulk autophagy especially in yeast. As similar to selective and non-selective autophagy, certain gens are required by mitophagy such as Atg1, Atg8 and Atg9 [30, 51]. The screening of several Atg mutants reveals that the Atg32 (the mitophagy receptor) and Atg33 genes, which are essential for Mitophagy play a direct role in controlling the mitophayge process $[52,53]$. In autophagic process, before starting the phagocytic process many cargos are delivered to degradative compartment of the cell. The cargos are processed by a complex of receptor protein in selective mode of autophagic process, supported by the recent research on autophagy. The quality control process in the mitochondria starts as a normal general autophagy process, it requires the same receptor protein complex to start and continue the process.

In this study we will present complexes of receptor proteins including components which form functional tetrad such as ligand, receptor, scaffold and an Atg family protein. Our current understanding of each of the four components and their interaction in the context of cargo selection process are considered in turn.

\section{ATG Family}

\subsection{ATG1, Early Signaling Events of Autophagy}

Among Atg family, autophagy-related protein 1 (Atg1) is the first player in the autophagy general process. In this issue, a signaling pathway linked two of the main regulators, the Atg1 and Atg9. Atg1 is responsible for the induction of auto-phagosome formation and Atg9, which is a transmembrane protein, which is thought to provide a membrane source by shuttled between the Golgi (pre-existing membrane sites).

Atg1 is responsible for the activation and phosphorylation of myosin light chain kinase which in turn activates myosin to drive transport of Atg9 [54]. During the general autophagy process a specialized double membrane vesicle, the autophagosomes, are formed in which the cell consumes its own contents and this is important for homeostasis and during cellular stress responses. These mechanisms of autophagy process include several steps such as induction, nucleation, and expansion of the auto-phagosome and fused with the lysosome to form the degradative auto-phagosome [55]. The early steps of autophagosomal degradation are controlled by a group of Atg genes, isolated from yeast. The signaling pathways depend upon Atg1 kinase and its associated proteins [56]. The target of Rapamycin kinase (TOR) which senses the availability of energy and nutrients for the regulation of cell growth and protein synthesis kept the Atg1 complex inactive by the process of phosphorylation [56]. Due to lack of nutrition TOR remain silent which results in the activation Atg1 complex and in turn autophagy. Two factors, class III PI3 kinase Vps34 and the Atg9 (a transmembrane protein), are needed downstream of Atg1 to initiate auto-phagosome formation: [55, 57]. In past, it was not clearly known how Atg1 activates autophagy since its substrates were members of its own complex [58].

\subsection{Atg8, an Autophagy-Related Ubiquitin-Like Protein}

Autophagy-related protein 8 (Atg8), an ubiquitin-like protein, plays key role in the formation of autophagosomal membranes. In eukaryotes the autophagy need the conjugation of Atg8 on side to side of autophagosomal membrane. Atg8 involved in autophagy process mediating the lysosome / vacuole-dependent turnover of the macromolecules and organelles [59]. It is important in macro-autophagy process, autophagosomes formation and maturation [60].

Like other Atg proteins, Atg8 is restricted to the PAS in cytoplasm under nutrient-rich conditions but during autophagy it associate with membrane. After this it localizes to the site of auto-phagosome nucleation [61]. The nucleation of the phagophore on PAS need class III phophoinositide 3-kinase complexes along with the accumulation of a set of Atg proteins. Vesicle expansion is believed to be triggered by the accumulation of Atg8 and other autophagy related proteins, particularly by providing the driving force for the formation of membrane curvature [62]. Atg8 protein is responsible for the expansion of phagophore when it conjugate to the membrane of phosphatidylethanolamine (PE). So, any mutations in it defect the formation of autophagosomes [63]. The amount of Atg8 protein is assumed to correlates quantitatively with the vesicle size and distributed on the both side of autophagosomes [64-67]. The vesicle expansion is followed by the fusion with lysosome. The Atg8 is either recycled by releasing from the membrane or gets degraded in the autolysosome.

Atg8 is present in two forms, the cytoplasmic and membrane associated [66]. The Atg8 is coupled to phosphatidylethanolamine (PE) (lipid constituent of plasma membrane) to achieve membrane association. This post translation modification (lipidation) is carried out by Atg8 conjugation system which comprise of cysteine protease ATG4 (capase family) and the proteins ATG3, ATG7 and ATG5-ATG12 complex [68]. Atg8 conjugation system is similar to the ubiquitination system that is Atg8 works in similar way to ubiquitin-like protein (Ubl), ATG7 works like E1 enzyme, ATG3 works like E2 enzyme and complex ATG12-ATG5 works like E3 ligase.

The last residues of the C-terminal of Atg8 cleaved by an Atg4 dependent post translational cleavage process that initiate the lipidation process. The PE is then coupled in several steps to C-terminal Gly116 that is exposed after the cleavage. In the 
first step the binding of Gly116 residues of Atg8 and cysteine residue of Atg7 occurred via thioester bond, this step depends upon the ATP. In the second step, the Atg8 is coupled with Atg3 via thioester linkage. Finally the Atg8 is coupled to the amine head of PE through amide bond immediately after detaching from Atg3. The ATG5-ATG12 complex plays a vital role in this final step [61].

Atg5 and Atg12 have been identified to be the part of Ubl conjugating system that promotes the ATG12-ATG5 conjugation through ATG7 and Atg10. This suggests that the ATG12-ATG8 conjugations are actually dependent.

ATG8 is also involved in different autophagy-related process such as Cytoplasm-to-vacuole targeting (Cvt) pathway [69]. The Cvt pathway is specific to the yeast which is responsible for the transportation of amino peptidase I (hydrolysis) to the yeast vacuole under nutrient rich condition. In the Cvt pathway, the formation of $\mathrm{Cvt}$ vesicles require the localization of Atg8 to the PAS which then fuse with the vacuole to deliver hydrolases necessary for degradation.

\subsection{Atg11, an Essential Player for the Regulation of Mitochondrial Degradation}

A cytosolic adapter protein, Autophagy related protein, is responsible for the recognition of selective cargo during autophagy process and being act as an adapter to link the mitophagy and Cvt pathway by directing the cargo to the autophagy machinery. During the Cvt pathway (which is an autophagy-related trafficking pathway), in this process, amino-peptidase I and alpha-mannosidase, are selectively transported from the cytoplasm to the lysosome-like vacuole. The cargo proteins Ams1 and Ape1 then form a complex with Atg19 that is recognized and by Atg11. Finally the cargo are directed by Atg11 to the pre-autophagosomal structure assembly site PAS along with actin cables [70] where the cargo is surrounded by the autophagosomes due to the emergence of isolation membrane [71]. Atg11 also regulate the mitochondrial degradation, confirming the presence of receptor protein for mitophagy that correspond to the Atg19 [37]. Atg32 has been recognized as receptor protein during the genetic screen for mutant yeast which is defective in mitophagy [18, 19, 72].

\subsection{Atg32 is a Receptor for Recruiting Mitochondria to the Vacuole}

Autophagy-related protein 32 (Atg32), a mitochondrial anchored protein, required in starvation depend and post log phase in which it act as a mitophagy receptor [18]. The Atg32 is specific to mitophagy as it is not required by any type of selective and non-selective autophagy [18, 19]. Atg32 is a single spanning protein in the outer membrane of mitochondria which consist of 529 amino acids with a molecular mass of 58968 Dalton. The N-terminal domain of $\operatorname{Atg} 32$ is exposed from outer membrane of mitochondria towards the cytoplasm. Atg32 act as a receptor for mitochondria when mitophagy is induced and Atg32 interact with Atg11 to form Atg11 and Atg32 complex that recruit mitochondria towards the PAS $[19,72]$. The formation of complex of Atg11 and Atg32 increases under certain circumstances in which autophagy is being induced. From this we suggest that Atg32 act as a mitochondrial receptor protein and during mitophagy it binds to the Atg11 (and adaptor protein) $[19,72]$. The Atg32 has been discovered as a novel mitophagy receptor protein during the screening of genome of mutant yeast that is defective in mitophagy process. Being involved in bulk autophagy and Cvt pathway, it has been suggested that Atg32 is specific to mitophagy [18, 72]. During the mitochondrial recruitment step, the Atg32 uses its WxxI motif to interact with Atg8 which then bind to the Atg11 to form Atg8 and Atg32 complex. The Atg32 and Atg8 complex act as cargo receptor because the efficiency of sequestration of mitochondria by isolation membrane increases with the formation of Atg32 and Atg8 complex [72]. The N-terminal domain of Atg32 (that is exposed toward the cytoplasm) has a tetra peptide sequence WQAI that is used by Atg32 for the interaction with Atg8 to efficiently activate mitophagy process. Another tetra peptide sequence $\mathrm{WxxL}$ is known to be important in Atg8.LC3 binding during selective autophagy and also found in the Cvt receptors Atg19 and p62/SQSTM1. Hence the mechanism of mitophagy is similar to the Cvt pathway, so, it is suggested that Atg32 is a mitochondrial surface receptor for selective mitophagy [73].

\section{Mitophagy is regulated by Nutrient Conditions}

Mitochondria are the power house of living cell as it is the main source of energy for the all process occurring in cell. In yeast cell the richest source of carbon is mitochondria. During the abnormal Nutrient Conditions, Mitophagy is induced to obtain the necessary carbon source to make mitochondria non-essential but blocked when the function of these organelles is needed for metabolism of a particular carbon source, in that case, Mitophagy is controlled separately from the bulk macro-autophagy, to observe that, yeast cells are submitted to nitrogen starvation after growth on different carbon sources, Mitophagy is induced when cells are cultured first with lactate as a main carbon source (YPL), and then shifted to amino acid starvation medium supplemented with glucose; however, the mitochondrial degradation process is blocked while lactate being the only carbon source. Under both conditions, macro-autophagy process is strongly activated. This finding supports the idea that mitochondria are degraded selectively.

In the yeast the number of mitochondria is regulated by its biosynthesis, segregation and degradation. Mitophagy is not a process which only eliminates the damaged mitochondria but also it is also involved in regulation of total number of mitochondria in a cell [14, 33, and 34].

During the mid-log growth phase of the cell, even if cells are shifted from YPL medium to glucose medium (YPD), Mitophagy is hardly detected. This is due to the decreased amount of mitochondria by segregation into daughter cells. 
In comparison, under nitrogen starvation conditions in the presence of glucose, Mitophagy could be detected. In this case, mitochondrial segregation process will be probably blocked by cell cycle arrest, and some amount of mitochondria that are not needed or required for fermentative growth degraded by Mitophagy. This idea can explain the induction of Mitophagy process that occurs at the post-log to stationary phase in lactate medium. During the cell stationary phase, the cell energy requirement will be reduced, and the cell requirement for mitochondria will decrease accordingly. Because mitochondrial segregation process is also stopped at this growth phase, Mitophagy process can be induced to maintain the cell to adapt these conditions and reduce the amount of the excessive mitochondria.

\section{Regulation of Mitophagy in Yeast}

The degradation of mitochondria via mitophagy is thought to be firmly regulated in a controlled manner showing the importance of this organelle in many processes ranging from energy production to cell death. However, the mechanism of molecular process through which autophagy machinery selects and degrades mitochondria is discovered but the upstream signaling pathway is still unraveled. The two kinases proteins, (mitogen activated protein kinases MAPKs) which are involved in the signaling pathway to induce mitophagy, are Slt2 and Hog1 [74, 75]. All the protein kinases that are involved in the Slt2 signaling pathway are MAPKKK (Bck1), MAPKK (Mkk1/Mkk2) and Wsc1 [74]. $\mathrm{Pbs} 2-\mathrm{Hog} 1$ and upstream stress sensor Sln1 are required for mitophagy in Hog1 signaling pathway [74]. Conversely the proteins involved in downstream of both pathways have not been discovered yet.

But the role of Slt2 is, however, explained in two ways: first under nitrogen starvation, when cells-induced Mitophagy is deficient in slt2-deleted cells [74], and second, the normal Mitophagy in slt2-deleted cells cultured to the post-log phase [76]. But the Slt2-related signaling pathway might be only associated with starvation-induced Mitophagy.

On the other hand, the phosphorylation of Ser114 and Ser119 on Atg32 do not occur efficiently during nitrogen starvation that is necessary for the formation of Atg32-Atg11 complex and mitophagy [75]. Similarly in Pichia pastoris the phosphorylation of Ser112 on PpAtg30 is required for the complex formation of PpAtg30-PpAtg11 and pexophagy [19]. These processes revealed that the kinases and the localization of kinases with Atg32 and/or PpAtg30 regulate both the pexophagy and mitophagy. Yet those kinases which directly phosphorylate Atg32 or PpAtg30 have not been identified.

\section{Atg33, the Related Specific Protein}

Atg33 is specific to mitophagy that has been identified by screening the genome of yeast mutant which is defective in mitophagy process due to mutation [53]. Atg33 is localized in the outer membrane of mitochondria and play important role in mitophagy but is not involved in the Cvt pathway of macro-autophagy or pexophagy. In the yeast strain when atg33 is knocked out, the process of mitophagy is partially inhibited when subjected to starvation but during the stationary phase the process of mitophagy is completely blocked. Though the function of Atg33 is still unknown in the mitophagy but it seems to play a role the quality control process by being a factor for the selection or detection of damaged or aged mitochondria, especially when cells reach the stationary phase $[53,77]$.

\section{Whi2, Uth1, and Aup1 a Quality Control Checkpoint for Mitochondria}

Whi2, Uth1 and Aup1 have been discovered to be related with mitophagy in addition to $\operatorname{Atg} 32$ and $\operatorname{Atg} 33$ [12, 35]. Whi2, a stress response protein, is responsible to influence mitophagy via nutrient sensing pathways. In yeast it also control stress response and to a lesser extent it may influence autophagy [78]. The stress response protein Whi2 and the Ras/PKA (protein kinase A) signaling pathway are possibly linked to the regulation of Mitophagy [79]. The Loss of Whi2 results in a decreased of the response to stresses, for example nutrient limitation upon entering into the stationary phase [80]. The same case will be, if the loss of Whi2 protein function leads to a decreased in the response after inhibition of TOR. For Whi2, there is no direct dependency of mitophagy process, or to a lesser extent of general autophagy, on Whi2 and from the stress responding, demonstrating for the first time a role of Whi2 in both processes. Old data show that Whi2 positively modulates autophagic flux to control the stress in the cell, Whi2 interacts physically with the phosphatases Psr1 and Psr2, and the transcription factor Msn2, which regulate the general stress response [81]. To monitor the mitochondrial quality and control the cell stress, it is therefore possible that the induction of the autophagy, and mitophagy process, then is directly or indirectly regulated by the general stress response.

Uth1 is a mitochondrial protein involved in autophagic mitochondrial degradation, it is found during a genetic screen experiments which aim at identifying proteins which are mainly involved in the regulation of the yeast life span [82]. It is one of the mitochondrial outer membrane proteins, which is reported to be required for mitophagy induced by rapamycin or nitrogen starvation [12]. The absence of Uth1 does not have dramatic effects under normal growth conditions; it may induce a $15-25 \%$ decrease of the mitochondrial cytochromes as well as other mitochondrial enzymes, and an increase of growth yield [83]. Uth1 has a high metabolic efficiency function that exists when linked to a general but slight slowing down of mitochondrial general functions. These characteristics explain the extended life span and the modified response to oxidative stress [84].

Uth1 is also involved in the autophagic degradation process of the mitochondria induced by rapamycin or nitrogen starvation which supports this assumption. Uth1 
does not participate in the autophagic machinery directly because the biochemical fingerprints related to this process (ALP activation and API processing) are as active as those in wild-type [12]. Uth1 protein might be involved in the recognition of mitochondria by the autophagic machinery, but it may not be the only protein which is involved in this process. It may also play a role in the early selective degradation of the mitochondria with the help of other components, and that will build connections between the apoptosis and autophagy [85]. Aup1 protein, which is localized in the inner mitochondrial membrane space, is identified in the process of the screening for the homologs of protein phosphatase that interact with Atg1 (serine/threonine kinase) required for the autophagy process and suggested the need of the efficient of mitophagy to survive during the prolonged stationary phase culture in a medium containing lactate as the carbon source [35]. During the stationary phase culture, Aup1 protein is required for Rtg3 nuclear translocation [86] and this is sufficient to explain the involvement of Aup1 in mitophagy [35]. And this is the way that explains how the RTG response plays a role in the regulation of mitophagy. On the other hand, the deletion of RTG3, a transcription factor that is responsible for the retrograde signaling pathway, will cause a defect in the stationary phase of mitophagy and the deletion of AUP1 leads disturbance in the phosphorylation of Rtg3 under these conditions. This implies that the function of Aup1 in mitophagy may be regulation of Rtg3-dependent transcription [86].

Among all factors, cellular oxidative status is one of them, that is responsible for the induction of mitophagy process. Reported by Deffieu et al. that the cellular level of reduced glutathione was increased by $\mathrm{N}$-acetylcysteine that in turn prevent the mitophagy [87]. Okamoto et al. reported that $\mathrm{N}$-acetylcysteine treatment suppressed the expression of $\operatorname{Atg} 32$ and in turn it inhibits the process of mitophagy [18]. From these two perspectives, it has been suggested that cellular oxidative condition effect the expression Atg32 and mitophagy process. As damaged mitochondria are eliminated by mitophagy so, the cellular oxidative status, which is onset by the production of ROS by damaged mitochondria, is related to the induction of the Mitophagy process in the yeast cell.

\section{The Physiological Role of Mitophagy in Yeast}

One obstruction of mitochondrial ATP synthesis process is the process which generates reactive oxygen species (ROS) which can damage the mitochondria itself. Accordingly, appropriate quality control process to this organelle is important to maintain proper cellular homeostasis. When yeast cells in respiratory growth face nitrogen starvation, the cells initiate Mitophagy, degrade excess mitochondria, and suppress ROS production from mitochondria, and as a result for that, the cells escape the severe oxidative damage that causes mtDNA deletion. This process is very important for yeast cells in nature because the cells frequently shift their metabolism between fermentation and respiration (depending on the environment), and are always at risk of starvation [73].

For example, when yeast cells grow in glucose-rich conditions, such as on the surface of grapes, they ferment the glucose and release ethanol preferentially. When cells absorb glucose, they switch their metabolism process from fermentation to respiration, the aerobic usage of ethanol. This conversion is called the di-auxic shift process and it is frequently observed in nature. During the di-auxic shift, the mitochondria proliferate to increase the cellular respiration. If the cells then encounter starvation stress, for example, if the nutrients are washed away by rain (from the surface of grapes), mitophagy process then start and the cells minimize the number of mitochondria to a basal level to achieve cellular energy requirements.

Recently, it has been reported that bulk autophagy-deficient yeast cells accumulate high levels of ROS, and these ROS cause the loss of mtDNA during nitrogen starvation [88]. Although these phenotypes of bulking autophagy-incomplete cells are similar to those of mitophagy-incomplete cells, the process of ROS accumulation is totally different. In bulk autophagyincomplete cells, cellular ROS accumulate during nitrogen starvation because the cellular amino acid pool is heavily reduced, and the expression of mitochondrial respiratory proteins and the ROS waste proteins are suppressed [88]. The level of ROS accumulated in bulk autophagy- incomplete cells during nitrogen starvation is much higher than that in mitophagy-deficient or wild-type cells. Several lines of evidence demonstrated that mitophagy eliminate damaged mitochondria from yeast cell before leading to cell death. The conditional knockout of a gene fmc1, responsible for the folding of F1Fo-ATPase, encoding the Fcm1 protein leads to mitophagy under anaerobic condition [33]. Others evidences suggest that any disturbance to the $\mathrm{Mdm} 38$, a mitochondrial $\mathrm{K}+\mathrm{H}+$ exchanger, results in the osmotic changes and mitochondrial swelling that cause the degradation of those mitochondria by mitophagy [34]. Ethidium bromide when used to block the replication of mitochondrial DNA we can observe the rapid degradation of mitochondria via autophagy process [38]. These results show that induction of mitophagy and mitochondrial damage are related to each other. Until the Atg32 discovery, there was no direct evidence that autophagy selectively eliminates damaged mitochondria. When the physiological role of atg32 in mitophagy process in yeast has been the characterized by confers selectivity for mitochondrial, mitochondrial squeezes as a cargo and that is necessary for the recruitment of this organelle by the autophagy machinery for mitophagy [72]. Further analysis suggested that mitophagy induced in wild type cell by encountering the nitrogen starvation and eliminate the mitochondria that have proliferated during respiratory growth. As a result the production of ROS is suppressed. Conversely in that cell from which atg32 gene has been deleted, the excess amount of ROS was produce by un-degraded 
mitochondria which further cause damage to other mitochondria and in turn more ROS produced by the resultant damage mitochondria. This lead to a condition called

Further analysis reveals that, when wild-type cells encounter nitrogen starvation, they induce mitophagy and quickly eliminate mitochondria that have proliferated during respiratory growth. As a result, cellular ROS production, which occurs mainly in mitochondria, is suppressed. On the other hand, in mitophagy-deficient atg32-deleted cells, undegraded mitochondria produce excess ROS during nitrogen starvation. ROS damage mitochondria, and damaged mitochondria produce further ROS, which finally leads to mtDNA deletion. Ultimately, cells with mtDNA deletion generate small colonies even in fermentable medium; this phenotype is called "petite" [73]. This suggests that mitophagy is required to regulate the number of mitochondria to minimize ROS production and, as a result, maintains the quality of mitochondria.

Several studies suggest that the interplay between mitochondria and autophagy. Bulk autophagy-incomplete yeast strains exhibited reduce mitochondrial membrane potential, activities of the electron transport chain, and higher levels of ROS and oxidative stress. This results in the loss of mtDNA [88]. In bulk autophagy- incomplete cells, cellular ROS accumulate during nitrogen starvation because the cellular amino acid pool is reduced and the expression of the ROS scavenger proteins is suppressed [88]. This finding suggests that autophagy, including mitophagy, contributes to the quality control of mitochondria.

In a contrasting situation, healthy mitochondria are required for efficient induction of autophagy under amino acid starvation [89]. Autophagic flux is regulated by Atg1, target of rapamycin (TOR) kinase complex I, and cAMP-dependent protein kinase A (PKA), whereas ATG8 induction is solely dependent on PKA. Defects in mitochondrial respiration induce PKA activity, resulting in the suppression of both ATG8 induction and autophagic flux. Therefore, mitochondrial dysfunction directly affects and regulates autophagy. The data presented by later indicate that defects in mitochondrial respiration inhibit autophagy including mitophagy during amino acid starvation. Consequently, the effect of mitochondrial dysfunction on the regulation of autophagy varies according to the severity of the defect. Furthermore, the inordinate accumulation of mitochondria is defective in respiration beyond a certain level which decreases the capacity for autophagy and mitophagy in these cells and evokes a negative feedback that results in cellular aging or death [89].

\section{Conclusion}

Mitochondria play essential roles in cell survival, it is required for all the critical function in the cell, starting from oxidative energy production till the crucial role in aging and programmed cell death, but all of that processes are controlled accurately.
One obstruction of mitochondrial ATP synthesis process is the process which generates reactive oxygen species (ROS) which can damage the mitochondria itself. Accordingly, appropriate quality control process to this organelle is important to maintain proper cellular homeostasis. When yeast cells in respiratory growth face nitrogen starvation, the cells initiate Mitophagy, degrade excess mitochondria, and suppress ROS production from mitochondria, and as a result for that, the cells escape the severe oxidative damage that causes mtDNA deletion. This process is very important for yeast cells in nature because the cells frequently shift their metabolism between fermentation and respiration (depending on the environment), and are always at risk of starvation.

We have reviewed the main proteins which play an essential role in controlling this process and the main pathways which lead to a highly controlled Mitophagy process. Mitochondrial degradation process is a high organized regulated process. When yeast cells are subjected to nitrogen starvation, degradation of mitochondria by autophagy occurs by both a selective process (Mitophagy) and a non-selective process.

In recent years, there has been a significant progress in the Mitophagy studies in yeast, the molecular processes and regulatory mechanisms have been well described, such as the specific Atg32-Atg11 interaction and the requirement for signaling by the two MAPKs Slt2 and Hog1, and the substantial role of Whi2, Uth1, and Aup1. Most of the Mitophagy-related genes identified in yeast are also present in mammals, it is reasonable to estimate that there will be similar molecular processes, regulatory mechanisms, and physiological roles in both yeast and mammals. These suggest that the molecular processes of Mitophagy are evolutionarily conserved. It is surprising then that the Mitophagy process and the essential factors identified to date are completely different between yeast and mammals. For example, in mammals, the mitochondrial receptor protein corresponding to Atg32 in yeast has not been identified.

\section{The Abbreviations used are}

$\begin{array}{ll}\text { Atg } & \text { Autophagy-related protein } \\ \text { PAS } & \text { phagophore-assembly site } \\ \text { PE } & \text { phosphatidylethanolamine } \\ \text { UbI } & \text { ubiquitin-like protein } \\ \text { MAPK } & \text { mitogen-activated protein kinases } \\ \text { ROS } & \text { Reactive Oxygen Species } \\ \text { ATG } & \text { Autophagy Gene } \\ \text { TOR } & \text { Targeting Of Rapamycin } \\ \text { PAS } & \text { Phagophore Assembly Site } \\ \text { YPL } & \text { Yeast extract, Peptone, Lactate } \\ \text { PKA } & \text { Protein Kinase A } \\ \text { mtDNA } & \text { Mitochondrial DNA }\end{array}$

\section{Acknowledgements}

We thank Dr.Houjin Zhang, Mr. Muhammad Junaid in the Department of Biotechnology, College of Life Science and 
Technology, Huazhong University of Science and Technology for technical assistance and helpful discussion.

\section{References}

[1] Kiel, J.A., Autophagy in unicellular eukaryotes. Philos Trans R Soc Lond B Biol Sci, 2010. 365(1541): p. 819-30.

[2] Yu, Z.Q., et al., Dual roles of Atg8-PE deconjugation by Atg4 in autophagy. Autophagy, 2012. 8(6): p. 883-92.

[3] Cabrera, S., et al., Autophagy, proteases and the sense of balance. Autophagy, 2010. 6(7): p. 961-3.

[4] Suzuki, K. and Y. Ohsumi, Molecular machinery of auto-phagosome formation in yeast, Saccharomyces cerevisiae. FEBS Lett, 2007. 581(11): p. 2156-61.

[5] Devenish, R.J. and D.J. Klionsky, Autophagy: mechanism and physiological relevance 'brewed' from yeast studies. Front Biosci (Schol Ed), 2012. 4: p. 1354-63.

[6] Nakatogawa, H., et al., Dynamics and diversity in autophagy mechanisms: lessons from yeast. Nat Rev Mol Cell Biol, 2009. 10(7): p. 458-67.

[7] Rubinsztein, D.C., T. Shpilka, and Z. Elazar, Mechanisms of auto-phagosome biogenesis. Curr Biol, 2012. 22(1): p. R29-34.

[8] Mizushima, N., Autophagy: process and function. Genes Dev, 2007. 21(22): p. 2861-73.

[9] Clark, S.L., Jr., Cellular differentiation in the kidneys of newborn mice studies with the electron microscope. J Biophys Biochem Cytol, 1957. 3(3): p. 349-62.

[10] Takeshige, K., et al., Autophagy in yeast demonstrated with proteinase-deficient mutants and conditions for its induction. J Cell Biol, 1992. 119(2): p. 301-11.

[11] Kissova, I., et al., Selective and non-selective autophagic degradation of mitochondria in yeast. Autophagy, 2007. 3(4): p. 329-36.

[12] Kissova, I., et al., Uth1p is involved in the autophagic degradation of mitochondria. J Biol Chem, 2004. 279(37): p. 39068-74.

[13] Cover, C., et al., Peroxynitrite-induced mitochondrial and endonuclease-mediated nuclear DNA damage in acetaminophen hepatotoxicity. J Pharmacol Exp Ther, 2005. 315(2): p. 879-87.

[14] Twig, G., et al., Fission and selective fusion govern mitochondrial segregation and elimination by autophagy. EMBO J, 2008. 27(2): p. 433-46.

[15] Twig, G., B. Hyde, and O.S. Shirihai, Mitochondrial fusion, fission and autophagy as a quality control axis: the bioenergetic view. Biochim Biophys Acta, 2008. 1777(9): p. 1092-7.

[16] Kim, I. and J.J. Lemasters, Mitophagy selectively degrades individual damaged mitochondria after photoirradiation. Antioxid Redox Signal, 2011. 14(10): p. 1919-28.

[17] Zhang, Y., et al., Adipose-specific deletion of autophagy-related gene 7 (atg7) in mice reveals a role in adipogenesis. Proc Natl Acad Sci U S A, 2009. 106(47): p.
19860-5.

[18] Okamoto, K., N. Kondo-Okamoto, and Y. Ohsumi, Mitochondria-anchored receptor Atg32 mediates degradation of mitochondria via selective autophagy. Dev Cell, 2009. 17(1): p. 87-97.

[19] Kanki, T., et al., Atg32 is a mitochondrial protein that confers selectivity during mitophagy. Dev Cell, 2009. 17(1): p. 98-109.

[20] Farre, J.C., et al., Phosphorylation of mitophagy and pexophagy receptors coordinates their interaction with Atg8 and Atg11. EMBO Rep, 2013.

[21] Motley, A.M., J.M. Nuttall, and E.H. Hettema, Atg36: the Saccharomyces cerevisiae receptor for pexophagy. Autophagy, 2012. 8(11): p. 1680-1.

[22] Motley, A.M., J.M. Nuttall, and E.H. Hettema, Pex3-anchored Atg36 tags peroxisomes for degradation in Saccharomyces cerevisiae. EMBO J, 2012. 31(13): p. 2852-68.

[23] Huang, W.P. and D.J. Klionsky, Autophagy in yeast: a review of the molecular machinery. Cell Struct Funct, 2002. 27(6): p. 409-20.

[24] Rock, K.L., et al., Inhibitors of the proteasome block the degradation of most cell proteins and the generation of peptides presented on MHC class I molecules. Cell, 1994. 78(5): p. 761-71.

[25] Lilienbaum, A., Relationship between the proteasomal system and autophagy. Int J Biochem Mol Biol, 2013. 4(1): p. 1-26.

[26] Gelino, S. and M. Hansen, Autophagy - An Emerging Anti-Aging Mechanism. J Clin Exp Pathol, 2012. Suppl 4.

[27] Wallace, D.C., A mitochondrial paradigm of metabolic and degenerative diseases, aging, and cancer: a dawn for evolutionary medicine. Annu Rev Genet, 2005. 39: p. 359-407.

[28] Damsky, C.H., Environmentally induced changes in mitochondria and endoplasmic reticulum of Saccharomyces carlsbergensis yeast. J Cell Biol, 1976. 71(1): p. 123-35.

[29] Thorsness, P.E., Structural dynamics of the mitochondrial compartment. Mutat Res, 1992. 275(3-6): p. 237-41.

[30] Kim, I., S. Rodriguez-Enriquez, and J.J. Lemasters, Selective degradation of mitochondria by mitophagy. Arch Biochem Biophys, 2007. 462(2): p. 245-53.

[31] Beaulaton, J. and R.A. Lockshin, Ultrastructural study of the normal degeneration of the intersegmental muscles of Anthereae polyphemus and Manduca sexta (Insecta, Lepidoptera) with particular reference of cellular autophagy. J Morphol, 1977. 154(1): p. 39-57.

[32] Campbell, C.L. and P.E. Thorsness, Escape of mitochondrial DNA to the nucleus in yme1 yeast is mediated by vacuolar-dependent turnover of abnormal mitochondrial compartments. J Cell Sci, 1998. 111 ( Pt 16): p. 2455-64.

[33] Priault, M., et al., Impairing the bioenergetic status and the biogenesis of mitochondria triggers mitophagy in yeast. Cell Death Differ, 2005. 12(12): p. 1613-21.

[34] Nowikovsky, K., et al., Mdm38 protein depletion causes loss of mitochondrial $\mathrm{K}+/ \mathrm{H}+$ exchange activity, osmotic swelling and mitophagy. Cell Death Differ, 2007. 14(9): p. 1647-56. 
[35] Tal, R., et al., Auplp, a yeast mitochondrial protein phosphatase homolog, is required for efficient stationary phase mitophagy and cell survival. J Biol Chem, 2007. 282(8): p. $5617-24$.

[36] Abeliovich, H., Mitophagy: the life-or-death dichotomy includes yeast. Autophagy, 2007. 3(3): p. 275-7.

[37] Kanki, T. and D.J. Klionsky, Mitophagy in yeast occurs through a selective mechanism. J Biol Chem, 2008. 283(47): $p$ 32386-93.

[38] Zhang, Y., et al., The role of autophagy in mitochondria maintenance: characterization of mitochondrial functions in autophagy-deficient S. cerevisiae strains. Autophagy, 2007. 3(4): p. 337-46.

[39] Sakai, Y., et al., Pexophagy: autophagic degradation of peroxisomes. Biochim Biophys Acta, 2006. 1763(12): p. 1767-75.

[40] Camougrand, N., et al., Uthlp: a yeast mitochondrial protein at the crossroads of stress, degradation and cell death. FEMS Yeast Res, 2004. 5(2): p. 133-40.

[41] DiMauro, S., Mitochondrial diseases. Biochim Biophys Acta, 2004. 1658(1-2): p. 80-8.

[42] Kundu, M. and C.B. Thompson, Macro-autophagy versus mitochondrial autophagy: a question of fate? Cell Death Differ, 2005. 12 Suppl 2: p. 1484-9.

[43] Skulachev, V.P., Bioenergetic aspects of apoptosis, necrosis and mitoptosis. Apoptosis, 2006. 11(4): p. 473-85.

[44] Narendra, D., et al., Parkin is recruited selectively to impaired mitochondria and promotes their autophagy. J Cell Biol, 2008. 183(5): p. 795-803

[45] Jin, S., p53, Autophagy and tumor suppression. Autophagy, 2005. 1(3): p. 171-3.

[46] Lemasters, J.J., Selective mitochondrial autophagy, or mitophagy, as a targeted defense against oxidative stress, mitochondrial dysfunction, and aging. Rejuvenation Res, 2005. 8(1): p. 3-5.

[47] Fliss, M.S., et al., Facile detection of mitochondrial DNA mutations in tumors and bodily fluids. Science, 2000. 287(5460): p. 2017-9.

[48] Penta, J.S., et al., Mitochondrial DNA in human malignancy. Mutat Res, 2001. 488(2): p. 119-33.

[49] Reggiori, F. and D.J. Klionsky, Autophagosomes: biogenesis from scratch? Curr Opin Cell Biol, 2005. 17(4): p. 415-22.

[50] Ishihara, N., et al., Auto-phagosome requires specific early Sec proteins for its formation and NSF/SNARE for vacuolar fusion. Mol Biol Cell, 2001. 12(11): p. 3690-702.

[51] Farre, J.C., et al., PpAtg30 tags peroxisomes for turnover by selective autophagy. Dev Cell, 2008. 14(3): p. 365-76.

[52] Kondo-Okamoto, N., et al., Autophagy-related protein 32 acts as autophagic degron and directly initiates mitophagy. J Biol Chem, 2012. 287(13): p. 10631-8.

[53] Kanki, T., et al., A genomic screen for yeast mutants defective in selective mitochondria autophagy. Mol Biol Cell, 2009. 20(22): p. 4730-8.
[54] Tang, H.W., et al., Atg1-mediated myosin II activation regulates auto-phagosome formation during starvation-induced autophagy. EMBO J, 2011. 30(4): p. 636-51.

[55] Simonsen, A. and S.A. Tooze, Coordination of membrane events during autophagy by multiple class III PI3-kinase complexes. J Cell Biol, 2009. 186(6): p. 773-82.

[56] Jung, C.H., et al., mTOR regulation of autophagy. FEBS Lett, 2010. 584(7): p. 1287-95.

[57] Webber, J.L. and S.A. Tooze, New insights into the function of Atg9. FEBS letters, 2010. 584(7): p. 1319-1326.

[58] Di Bartolomeo, S., et al., The dynamic interaction of AMBRA1 with the dynein motor complex regulates mammalian autophagy. J Cell Biol, 2010. 191(1): p. 155-68.

[59] Ohsumi, Y., Molecular dissection of autophagy: two ubiquitin-like systems. Nat Rev Mol Cell Biol, 2001. 2(3): p. 211-6.

[60] Eskelinen, E.L., New insights into the mechanisms of macro-autophagy in mammalian cells. Int Rev Cell Mol Biol, 2008. 266: p. 207-47.

[61] Geng, J. and D.J. Klionsky, The Atg8 and Atg12 ubiquitin-like conjugation systems in macroautophagy. 'Protein modifications: beyond the usual suspects' review series. EMBO Rep, 2008. 9(9): p. 859-64.

[62] Mizushima, N., et al., Dissection of auto-phagosome formation using Apg5-deficient mouse embryonic stem cells. J Cell Biol, 2001. 152(4): p. 657-68.

[63] Xie, Z. and D.J. Klionsky, Auto-phagosome formation: core machinery and adaptations. Nat Cell Biol, 2007. 9(10): p. 1102-9.

[64] Huang, W.P., et al., The itinerary of a vesicle component, Aut $7 \mathrm{p} / \mathrm{Cvt} 5 \mathrm{p}$, terminates in the yeast vacuole via the autophagy/Cvt pathways. J Biol Chem, 2000. 275(8): p. 5845-51.

[65] Kabeya, Y., et al., LC3, a mammalian homologue of yeast Apg8p, is localized in auto-phagosome membranes after processing. EMBO J, 2000. 19(21): p. 5720-8.

[66] Kirisako, T., et al., Formation process of auto-phagosome is traced with Apg8/Aut7p in yeast. J Cell Biol, 1999. 147(2): p. 435-46.

[67] Xie, Z., U. Nair, and D.J. Klionsky, Atg8 controls phagophore expansion during auto-phagosome formation. Mol Biol Cell, 2008. 19(8): p. 3290-8.

[68] Ichimura, Y., et al., A ubiquitin-like system mediates protein lipidation. Nature, 2000. 408(6811): p. 488-92.

[69] Kim, J. and D.J. Klionsky, Autophagy, cytoplasm-to-vacuole targeting pathway, and pexophagy in yeast and mammalian cells. Annu Rev Biochem, 2000. 69: p. 303-42.

[70] Monastyrska, I., et al., Atg11 directs auto-phagosome cargoes to the PAS along actin cables. Autophagy, 2006. 2(2): p. 119-21.

[71] Shintani, T., et al., Mechanism of cargo selection in the cytoplasm to vacuole targeting pathway. Dev Cell, 2002. 3(6): p. 825-37. 
[72] Kanki, T. and D.J. Klionsky, Atg32 is a tag for mitochondria degradation in yeast. Autophagy, 2009. 5(8): p. 1201-2.

[73] Kurihara, Y., et al., Mitophagy plays an essential role in reducing mitochondrial production of reactive oxygen species and mutation of mitochondrial DNA by maintaining mitochondrial quantity and quality in yeast. J Biol Chem, 2012. 287(5): p. 3265-72.

[74] Mao, K., et al., Two MAPK-signaling pathways are required for mitophagy in Saccharomyces cerevisiae. J Cell Biol, 2011. 193(4): p. 755-67.

[75] Aoki, Y., et al., Phosphorylation of Serine 114 on Atg32 mediates mitophagy. Mol Biol Cell, 2011. 22(17): p. 3206-17.

[76] Manjithaya, R., et al., A yeast MAPK cascade regulates pexophagy but not other autophagy pathways. J Cell Biol, 2010. 189(2): p. 303-10

[77] Kanki, T., K. Wang, and D.J. Klionsky, A genomic screen for yeast mutants defective in mitophagy. Autophagy, 2010. 6(2): p. $278-80$.

[78] Mendl, N., et al., Mitophagy in yeast is independent of mitochondrial fission and requires the stress response gene WHI2. J Cell Sci, 2011. 124(Pt 8): p. 1339-50.

[79] Muller, M. and A.S. Reichert, Mitophagy, mitochondrial dynamics and the general stress response in yeast. Biochem Soc Trans, 2011.39(5): p. 1514-9.

[80] Radcliffe, P., et al., Deregulation of CLN1 and CLN2 in the Saccharomyces cerevisiae whi2 mutant. Yeast, 1997. 13(8): p. 707-15.
[81] Kaida, D., et al., Yeast Whi2 and Psr1-phosphatase form a complex and regulate STRE-mediated gene expression. Genes Cells, 2002. 7(6): p. 543-52.

[82] Austriaco, N.R., Jr., Review: to bud until death: the genetics of ageing in the yeast, Saccharomyces. Yeast, 1996. 12(7): p. 623-30.

[83] Camougrand, N.M., et al., The "SUN" family: UTH1, an ageing gene, is also involved in the regulation of mitochondria biogenesis in Saccharomyces cerevisiae. Arch Biochem Biophys, 2000. 375(1): p. 154-60.

[84] Camougrand, N. and M. Rigoulet, Aging and oxidative stress: studies of some genes involved both in aging and in response to oxidative stress. Respir Physiol, 2001. 128(3): p. 393-401.

[85] Ogier-Denis, E. and P. Codogno, Autophagy: a barrier or an adaptive response to cancer. Biochim Biophys Acta, 2003. 1603(2): p. 113-28.

[86] Journo, D., A. Mor, and H. Abeliovich, Aup1-mediated regulation of Rtg3 during mitophagy. J Biol Chem, 2009. 284(51): p. 35885-95.

[87] Deffieu, M., et al., Glutathione participates in the regulation of mitophagy in yeast. J Biol Chem, 2009. 284(22): p. 14828-37.

[88] Suzuki, S.W., J. Onodera, and Y. Ohsumi, Starvation induced cell death in autophagy-defective yeast mutants is caused by mitochondria dysfunction. PLoS One, 2011. 6(2): p. e17412.

[89] Graef, M. and J. Nunnari, Mitochondria regulate autophagy by conserved signalling pathways. EMBO J, 2011. 30(11): p. 2101-14. 